\title{
Low Loss and Highly Birefringent Hollow-Core Photonic Crystal Fiber
}

Roberts, P. John; Williams, D.P.; Mangan, Brian J.; Sabert, H.

Published in:

Proceedings ECOC 2006

Link to article, DOI:

10.1109/ECOC.2006.4801214

Publication date:

2006

Document Version

Publisher's PDF, also known as Version of record

Link back to DTU Orbit

Citation (APA):

Roberts, P. J., Williams, D. P., Mangan, B. J., \& Sabert, H. (2006). Low Loss and Highly Birefringent HollowCore Photonic Crystal Fiber. In Proceedings ECOC 2006 (pp. 1-2). IEEE.

https://doi.org/10.1109/ECOC.2006.4801214

\section{General rights}

Copyright and moral rights for the publications made accessible in the public portal are retained by the authors and/or other copyright owners and it is a condition of accessing publications that users recognise and abide by the legal requirements associated with these rights.

- Users may download and print one copy of any publication from the public portal for the purpose of private study or research.

- You may not further distribute the material or use it for any profit-making activity or commercial gain

- You may freely distribute the URL identifying the publication in the public portal

If you believe that this document breaches copyright please contact us providing details, and we will remove access to the work immediately and investigate your claim 


\title{
Low Loss and Highly Birefringent Hollow-Core Photonic Crystal Fiber
}

P. J. Roberts (1), D. P. Williams (2), B. J. Mangan (3) and H. Sabert (4)

1 : COM, Danish Technical University (DTU), DK-2800 Kgs. Lyngby, Denmark. pir@com.dtu.dk

2 : BlazePhotonics Ltd., dpw_1969@hotmail.com, 3 : COM•DTU and Crystal Fibre A/S, bjm@crystal-fibre.com, 4 : BlazePhotonics Ltd., hsabert@btopenworld.com

\begin{abstract}
A hollow-core photonic crystal fiber design is proposed which enables both low-loss and polarizationmaintained signal propagation. The design relies on an arrangement of antiresonant features positioned on the glass ring that surrounds the air core.
\end{abstract}

\section{Introduction}

Hollow core photonic crystal fibers (HC-PCFs), which guide light due to the presence of a photonic bandgap, enable high-power light delivery in a single spatial mode. Since very low nonlinearity is ensured by most of the light being confined to the hollow core, the mode area can be relatively small, a typical size being around $40 \lambda^{2}$ where $\lambda$ is the optical wavelength. This allows an extremely high power-density at the fiber output and also implies resilience to bendinduced mode coupling which would otherwise degrade the output beam quality, increase loss and potentially cause fiber damage.

In many applications a polarization-maintaining $\mathrm{HC}$ PCF is either preferred or required. The presence of uncontrolled birefringence or polarization mode dispersion (PMD) can severely limit the pulse compression attainable in a communication or other pulse-delivery system, and will also severely degrade the performance in interferometric applications.

Given the generic birefringence of fabricated $\mathrm{HC}-\mathrm{PCF}$ caused even by small symmetry-breaking geometrical perturbations, there is clearly the need to develop low-loss intentionally birefringent fibers which show a high degree of polarization maintenance. Low-loss guidance can be facilitated by the incorporation of anti-resonant features within the core-surround $[1,2]$. A non-birefringent antiresonant core-surround design, which is just a glass ring of appropriately chosen thickness, has been shown to substantially decrease the field intensity at the glass/air interfaces and therefore the loss [2]. A fabricated fiber which incorporates a core-surround with this geometry has shown losses as low as $1.2 \mathrm{~dB} / \mathrm{km}$ [1]. In the current paper, an alternative core-surround geometry is considered which incorporates a number of elliptical features on a thin core-surround ring, see Fig. 1. The elliptical form was chosen since it is approximates a shape which is compatible with the surface tension forces which act during the fiber draw if an appropriate fiber perform is used. The size of the localized features is tuned so that the composite coresurround geometry is antiresonant within the cladding band-gap at the air light-line. This form of core-

surround mimics an arrangement of ARROW waveguides which enclose a central core [3], although the influence of the connecting ring of glass needs to be taken into consideration.

(a)

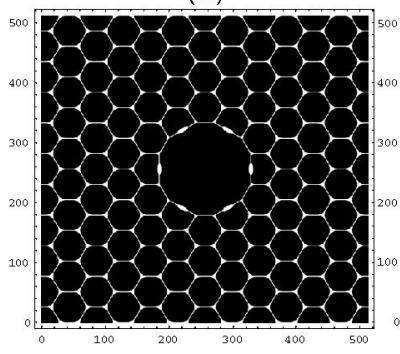

Fig. 1 Designs incorporating elliptical core-surround features. The fibre shown as (b) is birefringent.

Arrangements of the elliptical features which break 3fold rotational symmetry necessarily introduce birefringence. An example of such a design is given in Fig 1(b).

\section{Antiresonant core-surround geometry for low- loss propagation}

The dominant cause of loss in HC-PCFs is scattering from the roughness at the glass/air interfaces which results from an inherent thermodynamic attribute of the glass transition [1]. A relative measure for comparing the loss properties of different designs is afforded by the normalized interface field intensity $F$, defined by

$$
\mathrm{F}=\left(\varepsilon_{0} / \mu_{0}\right)^{1 / 2} \oint_{\text {interfaces }} \mathrm{d} s|\mathbf{e}|^{2} / \int_{\mathrm{x} \text {-section }} \mathrm{d} S(\mathbf{e} \wedge \mathbf{h}) \cdot \hat{\mathbf{z}}
$$

where the line integration in the numerator is taken just to the air side of the interfaces.

$F$ is shown for two non-birefringent HC-PCF designs in Fig. 2. The quantity is plotted against normalized wavenumber $\mathrm{k} \Lambda$ over the bandgap range, where $\Lambda$ is the cladding pitch. The fibers are identical except in the core-surround geometry. Fiber $A$, which is shown in Fig. 1(a), incorporates elliptical features with majordiameter length $2 a=0.4 \Lambda$ and minor-diameter length $2 b=0.133 \Lambda$ on the core-surround ring which has a thickness $t=0.03 \Lambda$. The core-surround of fiber $B$ contains no elliptical features: it is simply a ring of thickness $t=0.03 \Lambda$. The cladding structure of the fibers was chosen to be representative of fabricated $\mathrm{HC}$ - 


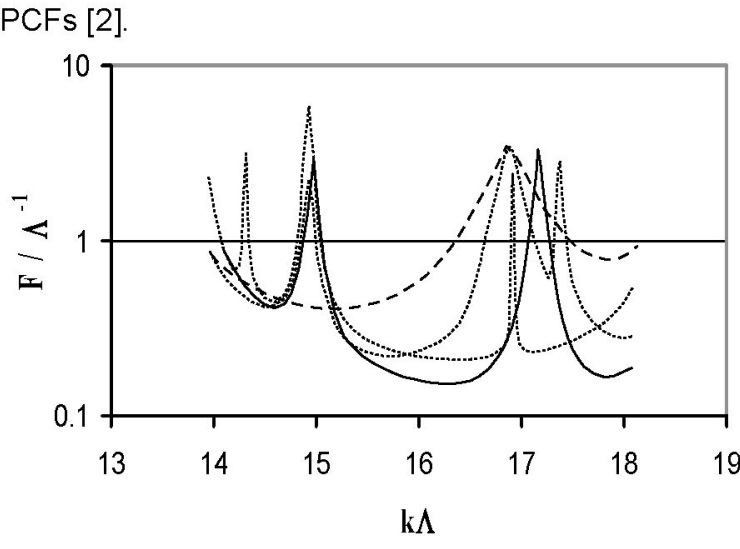

Fig. 2. The normalized interface field intensity, $F$, for a fiber with (continuous line) and without (dashed line) 6 elliptical core-surround inclusions. $F$ is also given for the two polarization modes of a birefringent fiber with 4 elliptical inclusions as dotted lines.

From Fig. 2, it is seen that the minimum attained $\mathrm{F}$ for fiber $A$ is about 3 times lower than for fiber $B$ in which the elliptical inclusions have been removed. This suggests fiber A should show lower loss than fiber B by a similar factor. The power in glass fraction for the two fibers is found to show a similar dependence as $F$, with the minimum value being about 2.2 times lower for fiber $A$. Hence the nonlinearity of fiber A will also be considerable less than that of fiber $B$.

The peaks in the traces of Fig. 2 are associated with anti-crossing events between the $\mathrm{HE}_{11}$-like core mode and modes of compatible symmetry associated with the core-surround glass arrangement. The incorporation of elliptical features introduces more anti-crossing events within the band gap range, but for the 7-cell core size, low-loss propagation is still possible over a wavelength range extending over nearly $200 \mathrm{~nm}$ centred on $1550 \mathrm{~nm}$. The number of unwanted anti-crossing events associated with the elliptical antiresonant core-surround inclusions is generically found to be fewer than is introduced by a continuously thick antiresonant core-surround ring such as the one considered in [2]

\section{Low-loss birefringent designs}

The simplest means of introducing birefringence to a fiber design which has localized features positioned on its core-surround ring is to remove one or more of them. Fig. 1(b) shows a fiber in which two opposing elliptical inclusions have been removed so that polarization degeneracy is lifted, but inversion and $180^{\circ}$ rotation symmetries remain. Apart from the removal of two elliptical features, the structure is identical to the fiber of Fig 1(a), results for which were given in the previous section.

Figure 2 shows $F$ for the two polarization channels (which correspond to degeneracy-split $\mathrm{HE}_{11}$-like modes) of the fiber of Fig. 1(b). It is seen that the two polarization states of the PM design show quite different mode anti-crossing features [4]. Although the minimum $F$ attained is a little higher than for the nonbirefringent reference form, it is around half the value for the corresponding non-birefringent form without antiresonant features within the core-surround.

Around a wavenumber of $k \Lambda=17.3$, one polarization mode shows a low F-value, whilst the other mode has a much higher F-value (and hence loss) since it is quite close to an anti-crossing. The birefringence, measured by the index difference between the polarization-split modes, becomes high near positions where one polarization mode undergoes anticrossing. At $k \Lambda=17.3$, this index difference is $5 \times 10^{-4}$. If a) this level of birefringence is sufficient to suppress cross-polarization scattering and b) random geometric perturbations do not cause a significant coupling of the low- $F$ polarization mode to the core-surround mode that is interacting with the high- $F$ mode, then the polarization mode with low $F$ will simultaneously show low-loss and high polarization maintenance.

\section{Conclusions}

Hollow core photonic crystal fiber designs which employ a number of localized features on the ring that surrounds the core can significantly reduce the field intensity at material/air interfaces as well as the fraction of the mode power which resides within the material. Thus such designs are candidates for showing low-loss and low nonlinearity. A core surround that incorporates a number of elliptical features of appropriate size and aspect ratio has been identified as a practical geometry with which to attain these desired properties, which manifest as the result of an antiresonance.

Birefringent designs have been considered which involve 2-fold symmetric arrangements of elliptical features on the core-surround ring. High birefringence is obtained when one polarization mode is close to anti-crossing with a mode associated with the coresurround whilst the other remains free of such an interaction. The interface field intensity of the polarization mode which is clear of mode anticrossing is maintained at a low level, which implies this polarization should show loss-loss if interpolarization mode coupling is maintained at a low level by the attendant high birefringence.

\section{References}

1 P. J. Roberts et al, Opt. Express, 13 (2005), 236

2 P. J. Roberts et al, Opt. Express, 13 (2005), 8277

3 N. M. Litchinitser et al, Opt. Lett., 27 (2002), 1592

4 F. Poletti et al, Opt. Express, 13 (2005), 9115 